\title{
Quantification of Activated Single-site Olefin Polymerization Catalysts on a Solid Support
}

\author{
Itzel Guerrero-Ríos, ${ }^{*}+, ;, *$ Elena Novarino, ${ }^{\dagger, \downarrow}$ Bart Hessen ${ }^{\dagger}$ and Marco W. Bouwkamp ${ }^{*} \dagger$ \\ ${ }^{\dagger}$ Molecular Inorganic Chemistry, Stratingh Insitute for Chemistry, University of Groningen, Nijenborgh 4, 9747 AG \\ Groningen, The Netherlands \\ ॠDutch Polymer Institute, POBox 902, 5600 AX Eindhoven, The Netherlands. \\ itzelgr@unam.mx,.mwbouwkamp@gmail.com
}

\section{CONTENTS:}

P2. Figure S1. Stability of $\left[p-\mathrm{HO}\left(\mathrm{C}_{6} \mathrm{H}_{4}\right) \mathrm{B}\left(\mathrm{C}_{6} \mathrm{~F}_{5}\right)_{3}\right]\left[\mathrm{Et}_{3} \mathrm{NH}\right]$ with trimethylalluminium (TMA) over time. $\left({ }^{19} \mathrm{~F}\right.$ NMR, toluene- $d_{8}$ )

P3. Figure $\mathrm{S} 2$. Stability of $\left[p-\mathrm{HO}\left(\mathrm{C}_{6} \mathrm{H}_{4}\right) \mathrm{B}\left(\mathrm{C}_{6} \mathrm{~F}_{5}\right)_{3}\right]\left[\mathrm{Et}_{3} \mathrm{NH}\right]$ with triisobutylalluminium (TIBA) over time. $\left({ }^{19} \mathrm{~F}\right.$ NMR, $\left.\mathrm{C}_{6} \mathrm{D}_{5} \mathrm{Br}\right)$.

P4. Figure S3. Chemisorption of $\left[p-\mathrm{HO}\left(\mathrm{C}_{6} \mathrm{H}_{4}\right) \mathrm{B}\left(\mathrm{C}_{6} \mathrm{~F}_{5}\right)_{3}\right]\left[\mathrm{Me}_{2} \mathrm{NPh}\right]$ on pacified silica over time. $\left({ }^{1} \mathrm{H} \mathrm{NMR}, \mathrm{C}_{6} \mathrm{D}_{5} \mathrm{Br}\right)$.

P4. Figure $\mathrm{S} 4 .{ }^{1} \mathrm{H}$ NMR of $\left(5\left[\mathrm{MeB}\left(\mathrm{C}_{6} \mathrm{~F}_{5}\right)_{3}\right]\right)$ in $\mathrm{C}_{6} \mathrm{D}_{5} \mathrm{Br}$.

P5. Figure S5. ${ }^{13} \mathrm{C}$ NMR of $\left(5\left[\mathrm{MeB}\left(\mathrm{C}_{6} \mathrm{~F}_{5}\right)_{3}\right]\right)$ in $\mathrm{C}_{6} \mathrm{D}_{5} \mathrm{Br}$.

P5. Figure S6. Immobilization of $\mathbf{1}$ with $\mathbf{N}^{\mathbf{P h}}$, trapping of activated species with allylmethyl thioether (AMT) and release in solution by ion exchange reaction with $\left[\mathrm{Bu}_{4} \mathrm{~N}\right] \mathrm{Br}$ for quantification in presence of internal standard $\mathrm{Cp}_{2} \mathrm{Fe}$. $\left({ }^{1} \mathrm{H}\right.$ NMR, $\left.\mathrm{C}_{6} \mathrm{D}_{6}\right)$.

P6. Figure S7. Immobilization of $\mathbf{1}$ with $\mathbf{N}^{\mathrm{Ph}}$, trapping of activated species with butenylmethyl thioether (BMT) and release in solution by ion exchange reaction with $\left[\mathrm{Bu}_{4} \mathrm{~N}\right] \mathrm{Br}$ for quantification in presence of internal standard $\mathrm{Cp}_{2} \mathrm{Fe}$. $\left({ }^{1} \mathrm{H} \mathrm{NMR}, \mathrm{C}_{6} \mathrm{D}_{5} \mathrm{Br}\right)$.

P7. Figure S8. Immobilization of $\mathbf{1}$ with $\mathbf{N}^{\mathrm{Ph}}$, trapping of activated species with AMT after $2.5 \mathrm{~h}$ and release in solution by ion exchange reaction with $[\mathrm{Bu} 4 \mathrm{~N}] \mathrm{Br}$ of a mixture of 4 and $\mathrm{Cp}^{*}{ }_{2} \mathrm{Zr}(\mathrm{Br})\left(2-\mathrm{C}_{6} \mathrm{D}_{4} \mathrm{Br}-\kappa \mathrm{C}\right)\left(\mathbf{8}-\boldsymbol{d}_{4}\right)$. $\left({ }^{1} \mathrm{H} \mathrm{NMR}\right.$, $\left.\mathrm{C}_{6} \mathrm{D}_{5} \mathrm{Br}\right)$.

P8. Figure S9. Immobilization of $\mathbf{1}$ with $\mathbf{N}^{\mathrm{Ph}}$, trapping of activated species with AMT after $2.5 \mathrm{~h}$ and release in solution by ion exchange reaction with $[\mathrm{Bu} 4 \mathrm{~N}] \mathrm{Br}$ of a mixture of 4 and $\mathrm{Cp}_{2}{ }_{2} \mathrm{Zr}(\mathrm{Br})\left(\mathrm{CH}_{2} \mathrm{SCH}_{2} \mathrm{CHCH}_{2}\right)(\mathbf{1 1})$. $\left({ }^{1} \mathrm{H}\right.$ NMR, toluene- $\left.d_{8}\right)$.

P9. Figure $\mathrm{S} 10 .{ }^{1} \mathrm{H}$ NMR of $\mathrm{Cp}^{*}{ }_{2} \mathrm{Zr}(\mathrm{Br})\{\mathrm{C}(\mathrm{Me}) \mathrm{NEt}-\kappa C, N\}(\mathbf{1 3})$ in $\mathrm{C}_{6} \mathrm{D}_{6}$.

P9. Figure $\mathrm{S} 11 .{ }^{13} \mathrm{C}$ NMR of $\mathbf{1 3}$ in $\mathrm{C}_{6} \mathrm{D}_{6}$.

P10. References 


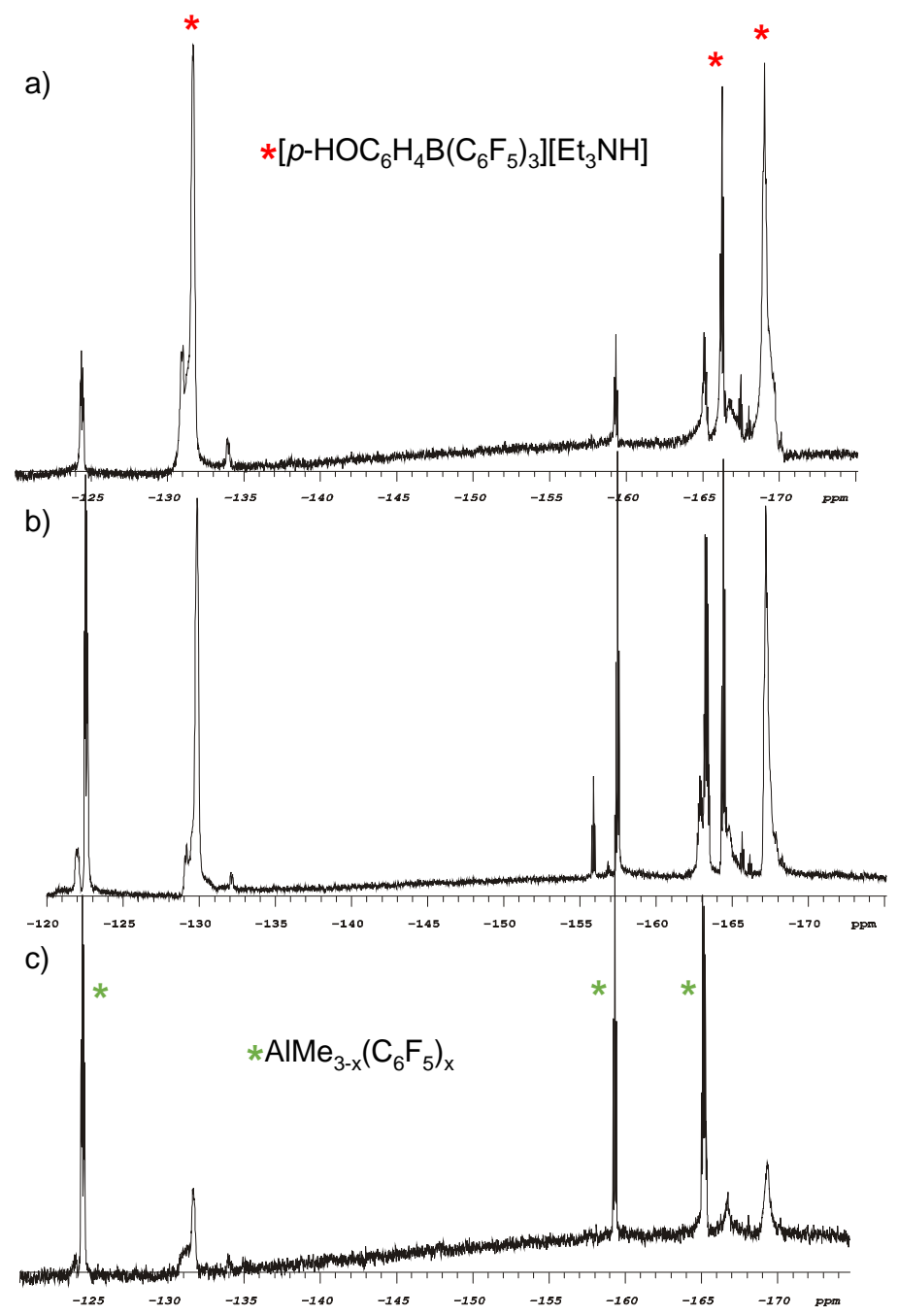

Figure S1. Stability of $\left[p-\mathrm{HO}\left(\mathrm{C}_{6} \mathrm{H}_{4}\right) \mathrm{B}\left(\mathrm{C}_{6} \mathrm{~F}_{5}\right)_{3}\right]\left[\mathrm{Et}_{3} \mathrm{NH}\right]$ with TMA over time by ${ }^{19} \mathrm{~F}$ NMR, $375 \mathrm{MHz}$, toluene- $d_{8}$ : a) 30 min; b) 2 hours; c) 8 hours. Identification based on reported spectroscopic data. ${ }^{1}$ 


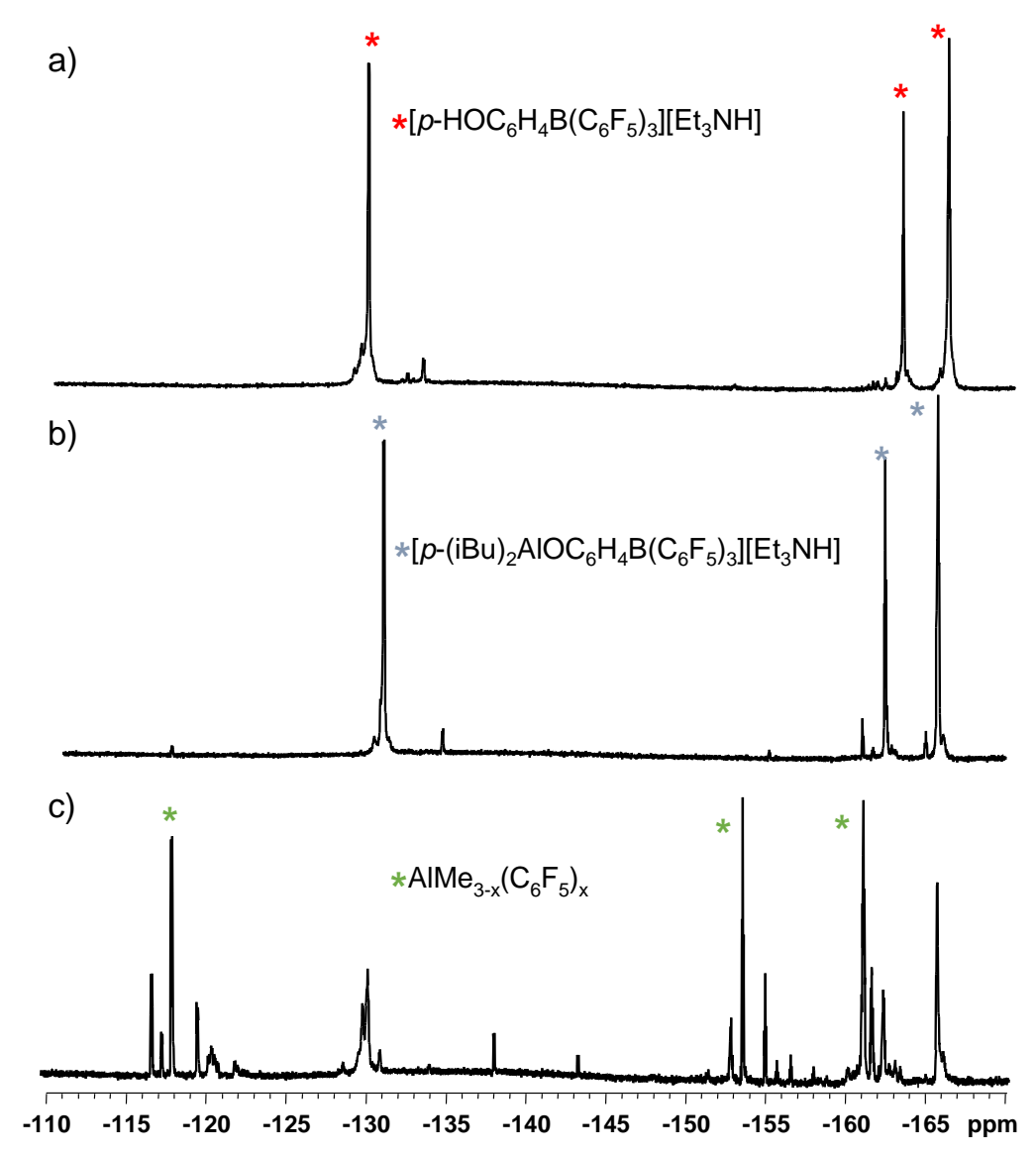

Figure S2. Stability of $\left[p-\mathrm{HOC}_{6} \mathrm{H}_{4} \mathrm{~B}\left(\mathrm{C}_{6} \mathrm{~F}_{5}\right)_{3}\right]\left[\mathrm{Et}_{3} \mathrm{NH}\right]$ with TIBA over time by ${ }^{19} \mathrm{~F} \mathrm{NMR}, 375 \mathrm{MHz}, \mathrm{C}_{6} \mathrm{D}_{5} \mathrm{Br}$ : a) 5 min; b) formation of $\left[p-\left(\mathrm{CH}_{2} \mathrm{CHMe}_{2}\right)_{2} \mathrm{AlO}\left(\mathrm{C}_{6} \mathrm{H}_{4}\right) \mathrm{B}\left(\mathrm{C}_{6} \mathrm{~F}_{5}\right)_{3}\right]\left[\mathrm{Et}_{3} \mathrm{NH}\right]$ after $30 \mathrm{~min}$; c) decomposition of $[p$ $\left.\left(\mathrm{CH}_{2} \mathrm{CHMe}_{2}\right)_{2} \mathrm{AlO}\left(\mathrm{C}_{6} \mathrm{H}_{4}\right) \mathrm{B}\left(\mathrm{C}_{6} \mathrm{~F}_{5}\right)_{3}\right]\left[\mathrm{Et}_{3} \mathrm{NH}\right]$ at $80^{\circ} \mathrm{C}$ for $3 \mathrm{~h}$. Identification based on reported spectroscopic data. ${ }^{2}$ 

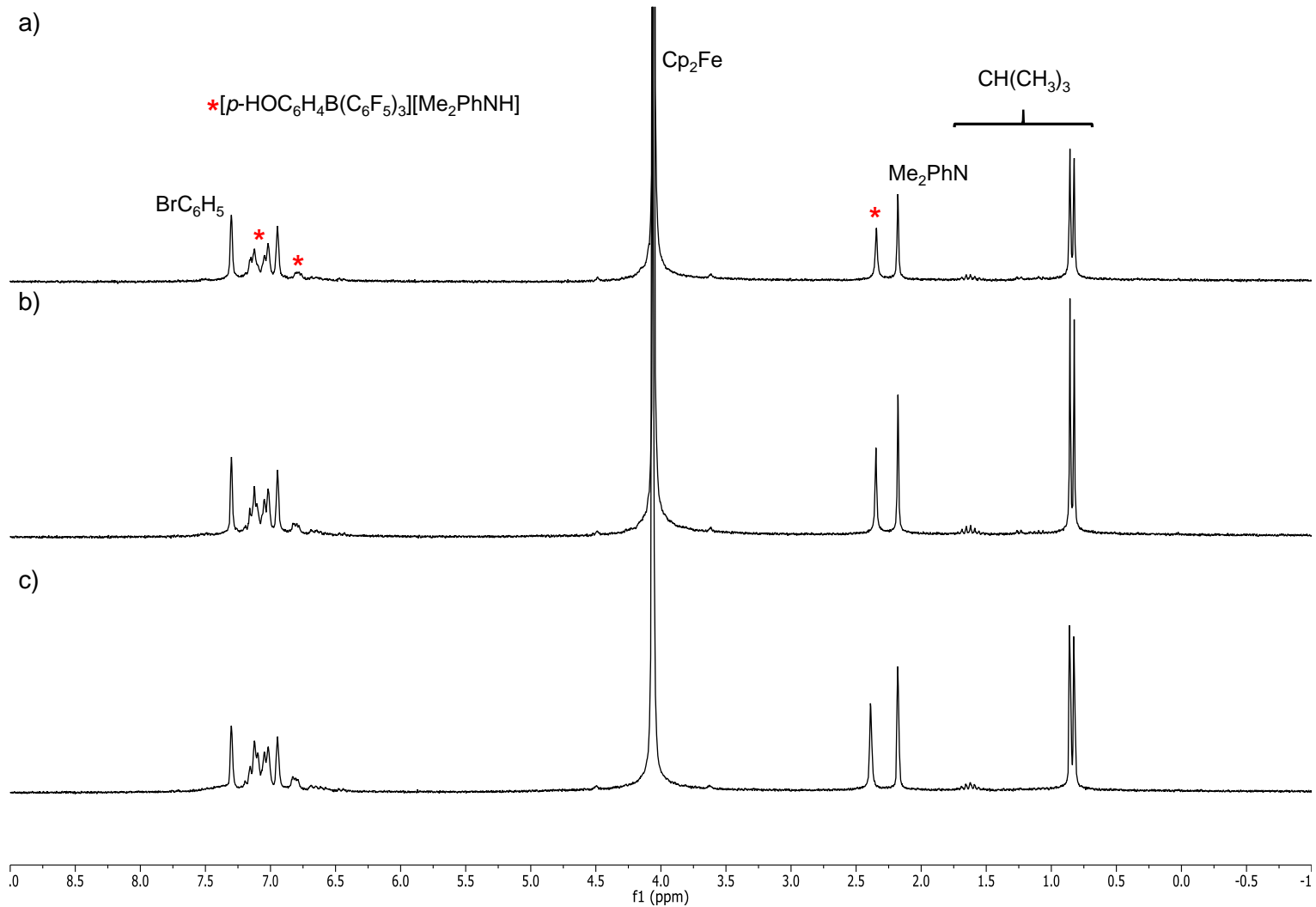

Figure S3. Chemisorption of $\left[p-\mathrm{HOC}_{6} \mathrm{H}_{4} \mathrm{~B}\left(\mathrm{C}_{6} \mathrm{~F}_{5}\right)_{3}\right]\left[\mathrm{Me}_{2} \mathrm{PhNH}\right]$ on $\mathrm{Al}(i \text { - } \mathrm{Bu})_{3}$-modified silica support by ${ }^{1} \mathrm{H}$ NMR, $400 \mathrm{MHz}, \mathrm{C}_{6} \mathrm{D}_{5} \mathrm{Br}$ : a) 15 min, b) 4 h, c) overnight.

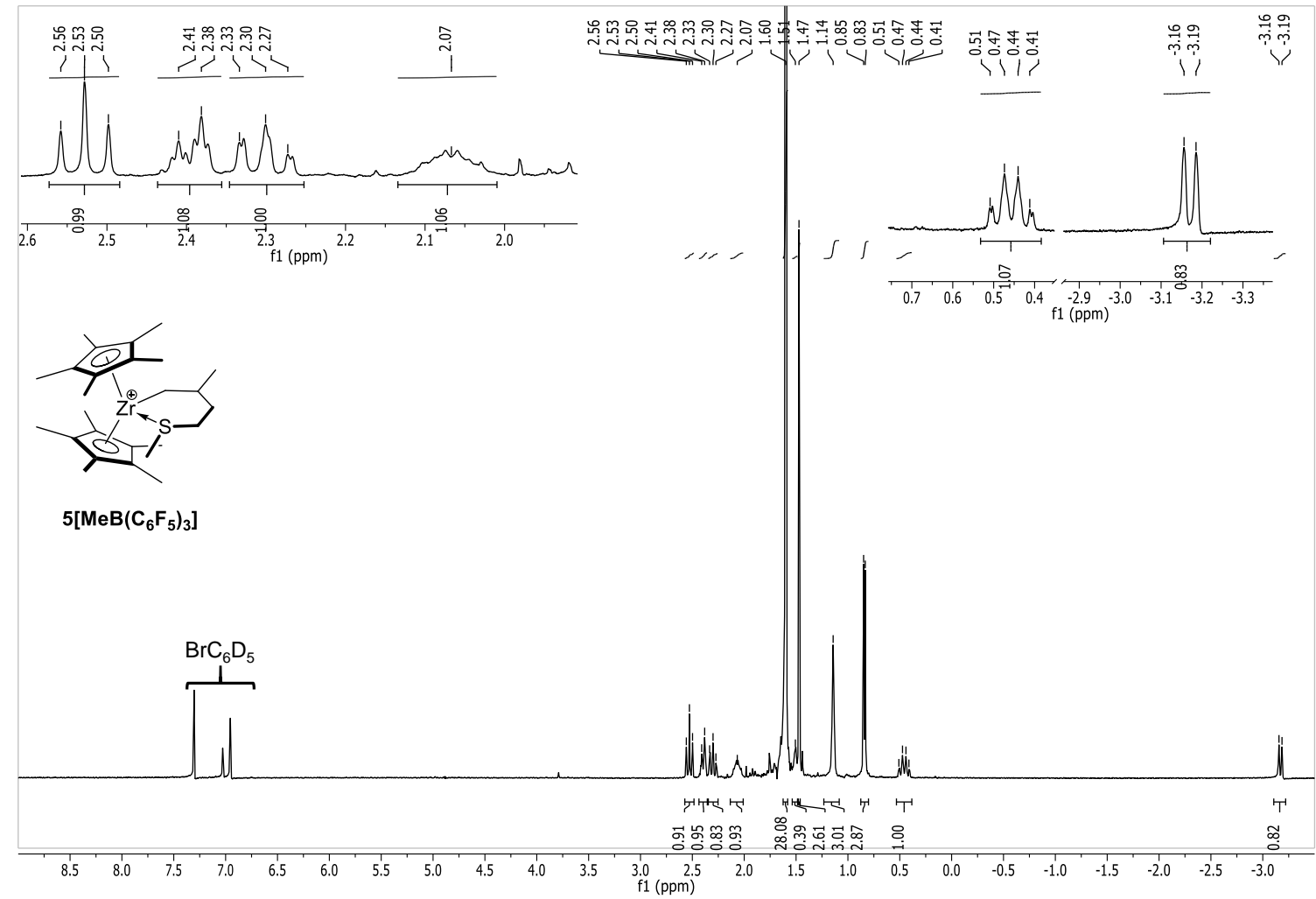

Figure S4. ${ }^{1} \mathrm{H}$ NMR $\left(500 \mathrm{MHz}, \mathrm{C}_{6} \mathrm{D}_{5} \mathrm{Br}\right)$ of $\left(\mathbf{5}\left[\mathrm{MeB}\left(\mathrm{C}_{6} \mathrm{~F}_{5}\right)_{3}\right]\right)$. 


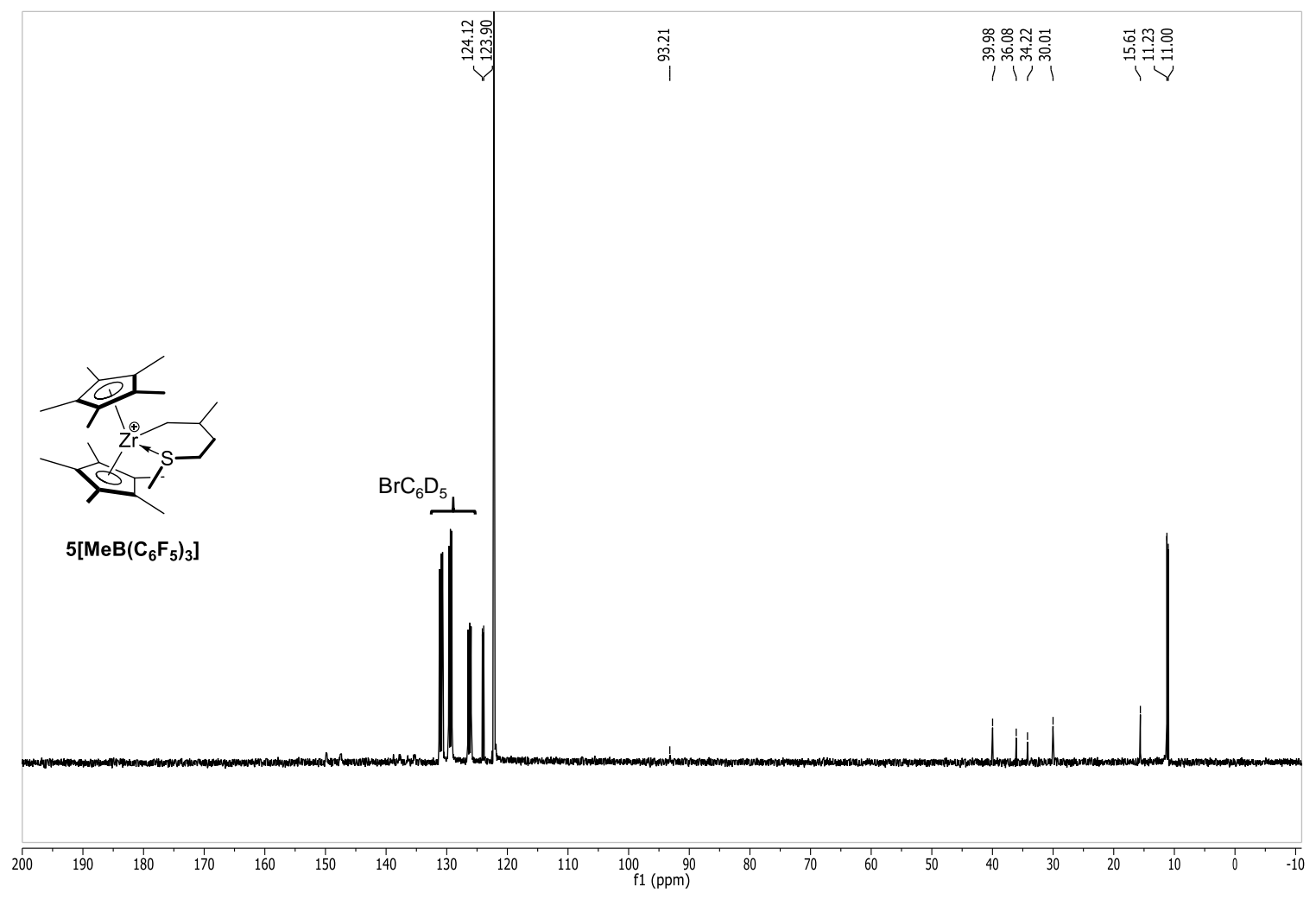

Figure S5. ${ }^{13} \mathrm{C}\{1 \mathrm{H}\}$ NMR $\left(125.7 \mathrm{MHz}, \mathrm{C}_{6} \mathrm{D}_{5} \mathrm{Br}\right)$ of $\left(\mathbf{5}\left[\mathrm{MeB}\left(\mathrm{C}_{6} \mathrm{~F}_{5}\right)_{3}\right]\right)$.

a)

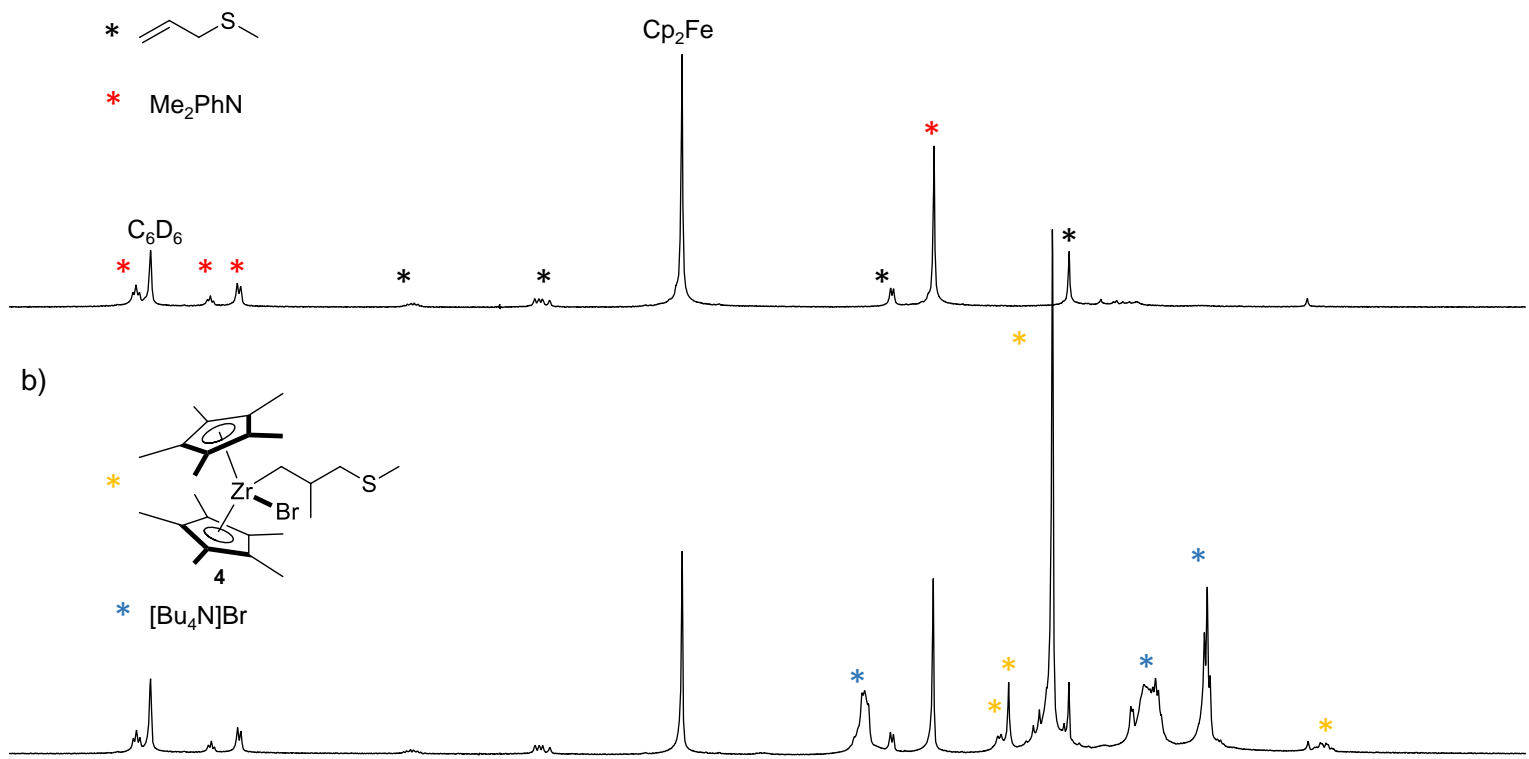

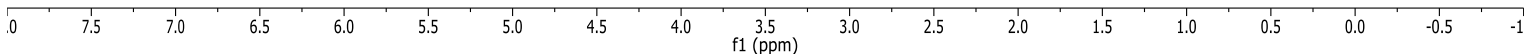

Figure S6. Immobilization study in presence of AMT by ${ }^{1} \mathrm{H} N M R, 300 \mathrm{MHz}, \mathrm{C}_{6} \mathrm{D}_{6}$ : a) immobilization of 1 with $\mathbf{N}^{\mathrm{Ph}}$, trapping with AMT; and, b) release in solution of $\mathbf{4}$ by reaction with $\left[\mathrm{Bu}_{4} \mathrm{~N}\right] \mathrm{Br}$ in presence of internal standard $\mathrm{Cp}_{2} \mathrm{Fe}$. Identification based on reported spectroscopic data. ${ }^{2}$ 


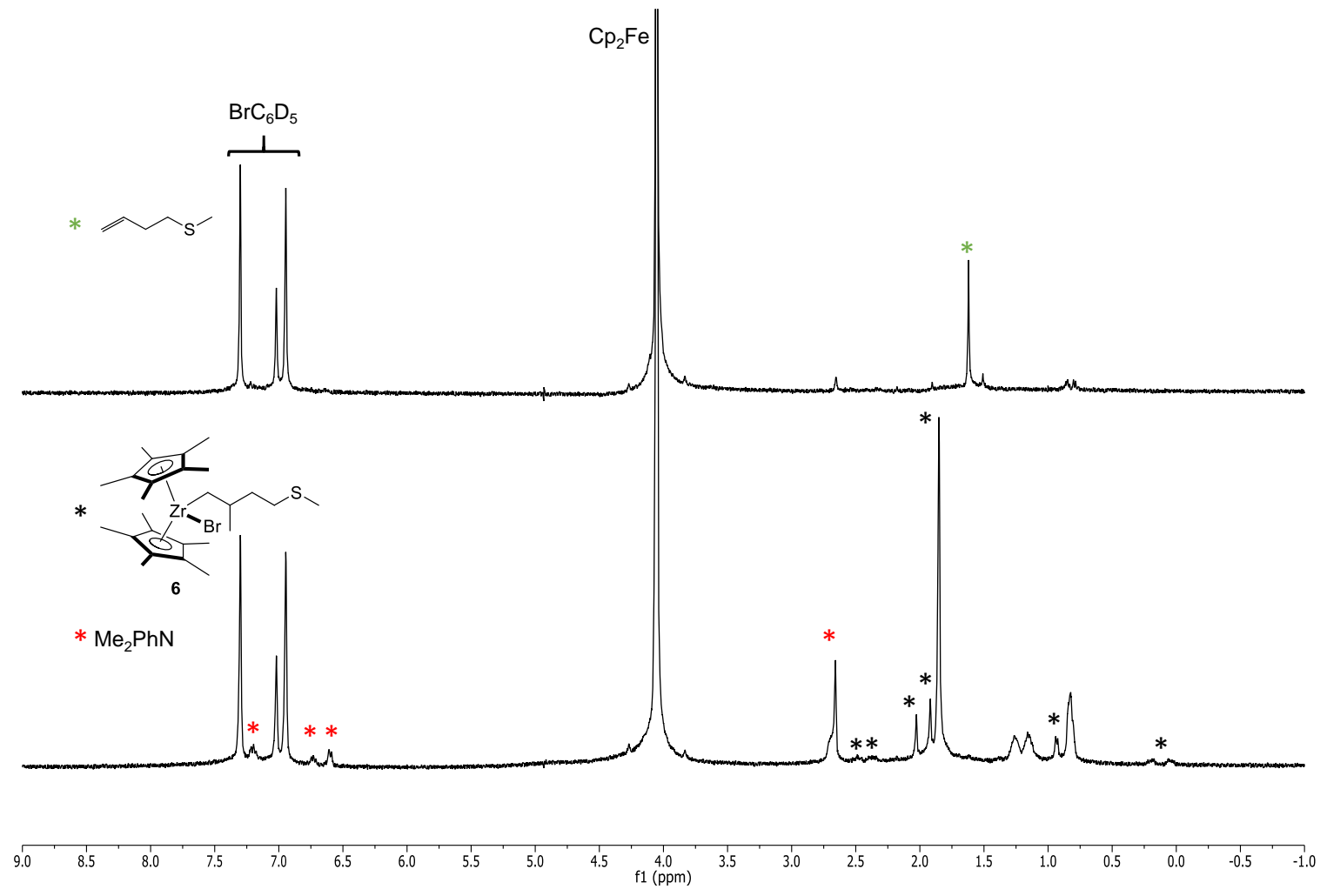

Figure S7. Immobilization study in presence of BMT by ${ }^{1} \mathrm{H}$ NMR, $300 \mathrm{MHz}, \mathrm{C}_{6} \mathrm{D}_{5} \mathrm{Br}$ : a) immobilization of 1 with $\mathbf{N}^{\mathbf{P h}}$, trapping with BMT; and, b) release in solution of $\mathbf{6}$ by reaction with $\left[\mathrm{Bu}_{4} \mathrm{~N}\right] \mathrm{Br}$ in presence of internal standard $\mathrm{Cp}_{2} \mathrm{Fe}$. Identification based on reported spectroscopic data. ${ }^{2}$ 
a)
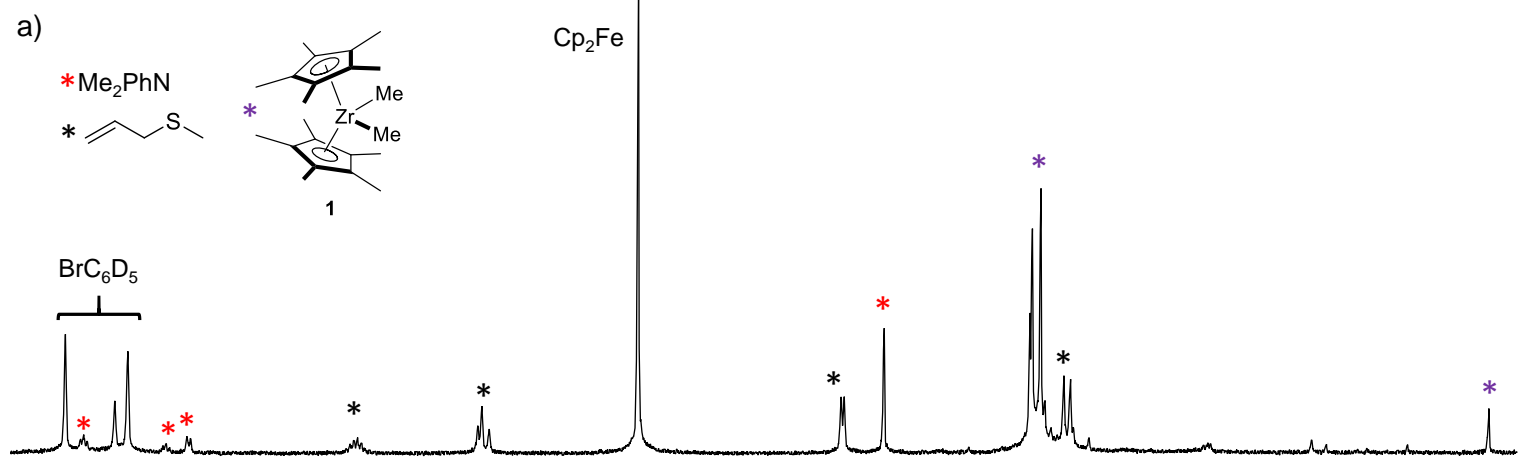

b)
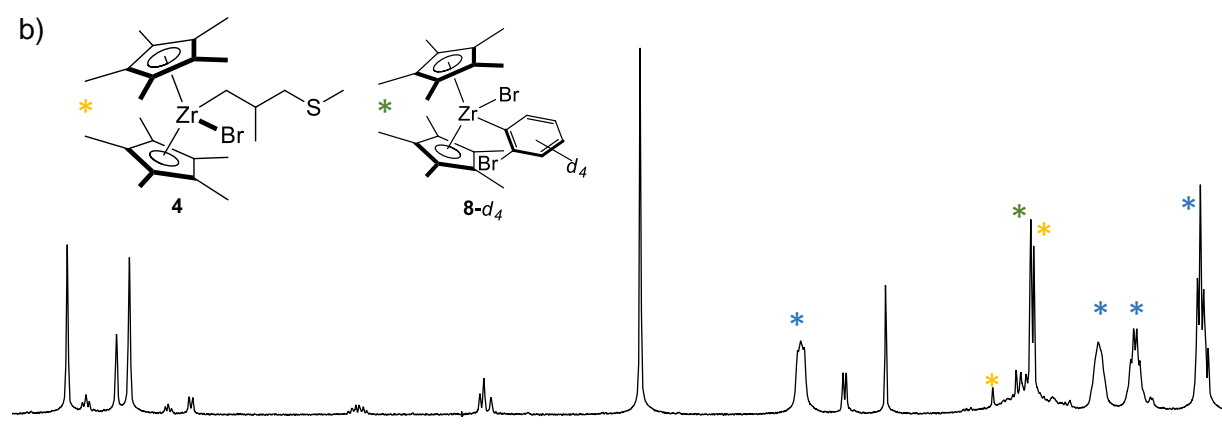

7.5
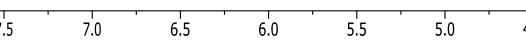

$\begin{array}{ll}3.5 & \\ \text { f1 (ppm) } & 3.0\end{array}$

Figure S8. Immobilization study with addition of AMT after $2.5 \mathrm{~h}$ by ${ }^{1} \mathrm{H}$ NMR, $300 \mathrm{MHz}, \mathrm{C}_{6} \mathrm{D}_{5} \mathrm{Br}$ : a) immobilization of $\mathbf{1}$ with $\mathbf{N}^{\mathrm{Ph}}$, trapping with AMT at $2.5 \mathrm{~h}$; and, b) release in solution of a mixture of $\mathbf{4}$ and 8- $\boldsymbol{d}_{\mathbf{4}}$ by reaction with $\left[\mathrm{Bu}_{4} \mathrm{~N}\right] \mathrm{Br}$ in presence of internal standard $\mathrm{Cp}_{2} \mathrm{Fe}$. Identification based on reported spectroscopic data. ${ }^{2}$ 


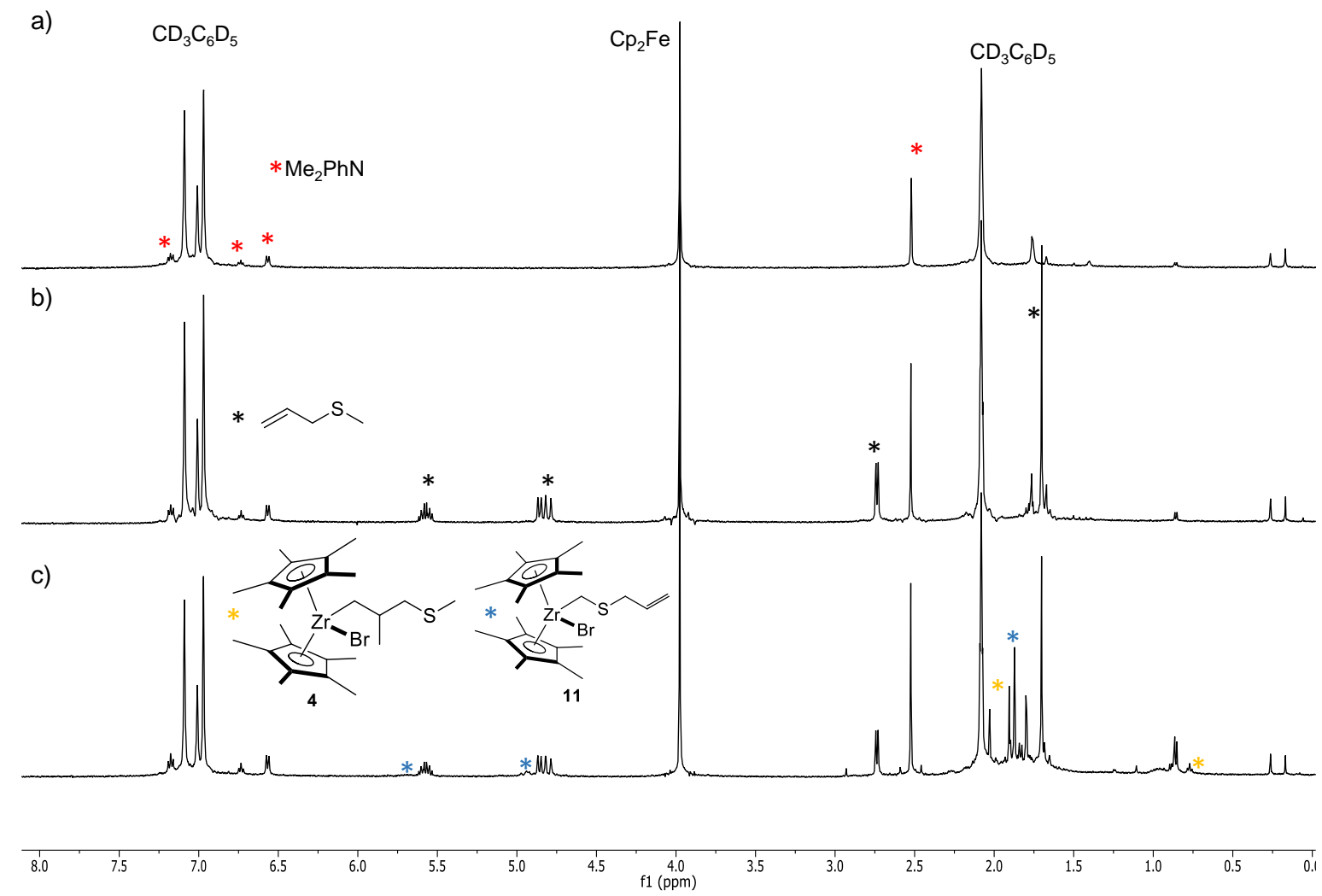

Figure S9. Immobilization study with addition of AMT after $2.5 \mathrm{~h}$ by ${ }^{1} \mathrm{H}$ NMR, $300 \mathrm{MHz}$, toluene- $d_{8}$ : a) immobilization of 1 with $\mathbf{N}^{\mathrm{Ph}}$, trapping with AMT at $2.5 \mathrm{~h}$; and, b) release in solution of a mixture of $\mathbf{4}$ and $\mathbf{1 1}$ by reaction with $\left[\mathrm{Bu}_{4} \mathrm{~N}\right] \mathrm{Br}$ in presence of internal standard $\mathrm{Cp}_{2} \mathrm{Fe}$. Identification based on reported spectroscopic data. ${ }^{2}$ 


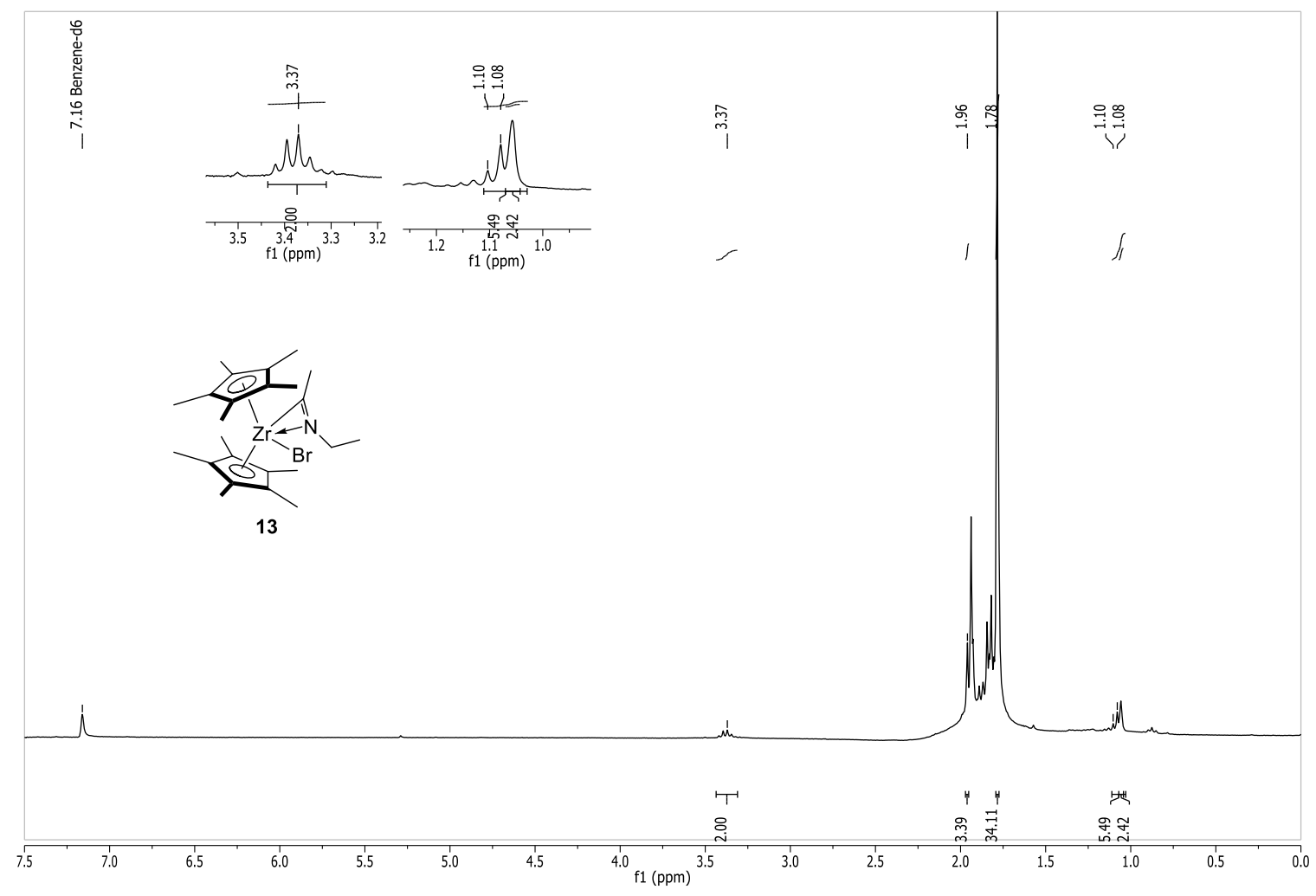

Figure S10. ${ }^{1} \mathrm{H}$ NMR $\left(300 \mathrm{MHz}, \mathrm{C}_{6} \mathrm{D}_{6}\right)$ of 13. Identification based on reported spectroscopic data. ${ }^{2}$

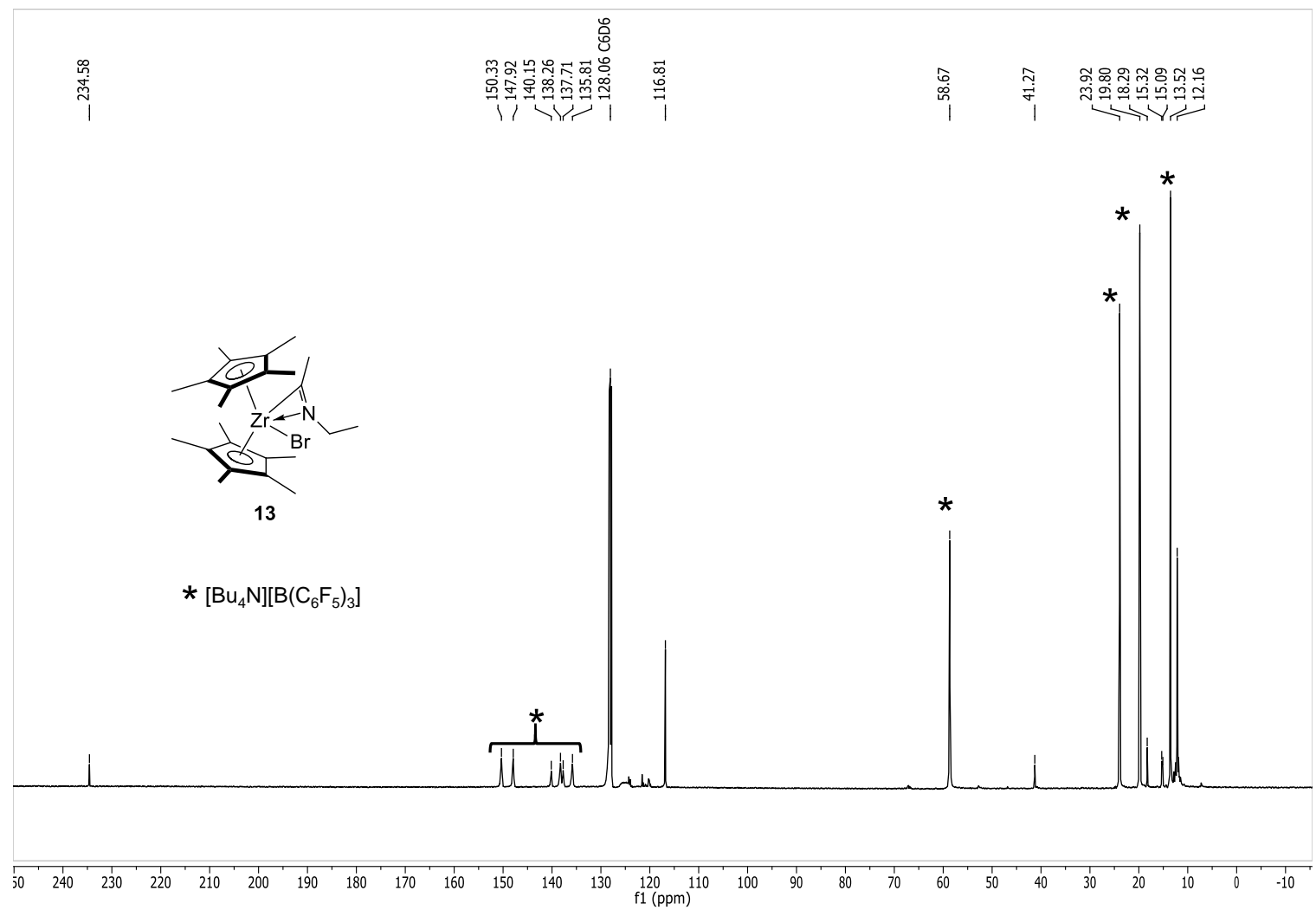

Figure S11. ${ }^{13} \mathrm{C}\left\{{ }^{1} \mathrm{H}\right\}$ NMR $\left(125 \mathrm{MHz}, \mathrm{C}_{6} \mathrm{D}_{6}\right)$ of 13. $\left[\mathrm{Bu}_{4} \mathrm{~N}\right]\left[\mathrm{B}\left(\mathrm{C}_{6} \mathrm{~F}_{5}\right)_{4}\right]$ present, $\delta: 150.4(o-\mathrm{CF}), 140.0(p-\mathrm{CF}), 138.0$ (m-CF), 124.1 (ipso-C), $58.3\left(\mathrm{NCH}_{2} \mathrm{CH}_{2} \mathrm{CH}_{2} \mathrm{CH}_{3}\right), 23.3\left(\mathrm{NCH}_{2} \mathrm{CH}_{2} \mathrm{CH}_{2} \mathrm{CH}_{3}\right), 19.4\left(\mathrm{NCH}_{2} \mathrm{CH}_{2} \mathrm{CH}_{2} \mathrm{CH}_{3}\right), 13.52$ $\left(\mathrm{NCH}_{2} \mathrm{CH}_{2} \mathrm{CH}_{2} \mathrm{CH}_{3}\right)$. Identification based on reported spectroscopic data. ${ }^{2}$ 


\section{REFERENCES:}

1. Bochmann, M.; Sarsfield, M.J. Organometallics 1998, 17, 5908-5912.

2. E. Novarino, PhD Thesis, University of Groningen, 2010. 